the acceptability and preferences for STI screening among a sample of pregnant, HIV-infected women at two clinics near Pretoria, South Africa.

Methods We approached potentially eligible HIV-infected pregnant women at their first antenatal care visit to invite them to receive STI screening that was integrated into the appointment. Trained staff collected data on STI screening preferences. Following enrollment, women were requested to provide two self-collected vaginal swabs for STI testing (GeneXpert, Cepheid, Sunnyvale, CA, USA).

Results Of the 171 women eligible for enrollment, 168 (98.2\%) agreed to participate in the STI study, including STI testing and self-collected vaginal swabs. Of the 167 complete responses, $15.6 \% \quad(n=26)$ preferred a urine test for STI screening, $41.1 \% \quad(n=69)$ vaginal swab, and $42.9 \% \quad(n=72)$ had no preference. Of those who preferred the urine test, the most common reasons were "ease" of the test $(n=20,12.0 \%)$, followed by anticipation of the test being "less painful" $(n=3$, $1.8 \%)$, "less uncomfortable" $(\mathrm{n}=2,1.2 \%)$, "less embarrassing" $(n=1,0.6 \%)$, and more familiar $(n=1,0.6 \%)$. However, when given directions on the vaginal swab collection procedures, all $168(100 \%)$ women complied and received successful test results.

Conclusion Integration of an STI screening protocol, including self-administered vaginal swabs, into first antenatal care visits appeared to be highly acceptable in this setting among HIVinfected pregnant women. Despite variability in preferences, all women adequately and satisfactorily self-collected vaginal samples, allowing for the timely detection and treatment of infections that may have been otherwise missed.

\section{P3.71 CORRELATES OF HIV INFECTION AMONG KENYAN WOMEN SCREENED FOR A INTRA-VAGINAL CONTRACEPTIVE RING STUDY IN KISUMU KENYA, 2015}

EM Makanga, B Nyagol, Oliver V Olilo G. Kenya Medical Research Institute, Kisumu, Kenya

\subsection{6/sextrans-2017-053264.306}

Introduction Women in Sub -Saharan africa continue to be dispropotionately affectced by the HIV epidemic. Correlates of HIV infection need to be better understood, and prevention strategies adapted, to account for risk patterns linked to particular settings or situations.

Methods We assessed prevalence and correlates of HIV infection among women 18-34 years of age screened for a contraceptive vaginal ring study. Women provided socio-demographic, medical information, and underwent real-time parallel rapid HIV testing and medical evaluation and sexually transmitted infections (STI) testing.

Results Of the 459 women screened, the median age was 25 (IQR: 21-28). Majority of whom were married (69\%), and more than a half $(69 \%)$ had primary or less level of education. Overall, HIV prevalence was $14.5 \%$, with the married and widowed recording a significantly higher burden of HIV (25\% and 50\%) respectively. Slightly more than a half $(55 \%)$ of HIV positive women perceived their health to be good. Women who tested positive for herpes simplex and gonorrhoea had significantly higher prevalence of HIV ( $(6 \%$ vs. $21 \%, \mathrm{p}<0.001 \%$ and $14 \%$ vs. $39 \% ; \mathrm{p}=0.003)$ respectively.
Women ages 25-29 years old had a 3 fold increased odds of HIV acquisition compared to $18-24$ year olds $(\mathrm{aOR}=3.61$; 95\% CI:1.23-10.49, $\mathrm{p}=0.019$ ), while those aged 30-34 had a 5 fold increased risk $(\mathrm{aOR}=5.36 ; 95 \% \mathrm{CI}: 1.23-19.60$, $\mathrm{p}=0.011)$. Widowed had nearly 6 times risk of HIV acquisition compared to single women $((\mathrm{aOR}=5.85$; 95\% CI:1.3924.51, $\mathrm{p}=0.016)$, whereas women reporting to have to have had sexual intercourse with partners of unknown HIV status in the last 3 months were four times more likely to test positive for HIV than those who did not (OR 4.10 95\% CI: 1.47-11.41).

Conclusion Behavioural, biological and structural factors continue to put women at risk of HIV.There is need for multipronged prevention strategies including female controlled multipurpose technologies to reduce their HIV burden.

\section{P3.72 THE PREVALENCE OF ANAL HIGH-RISK HUMAN PAPILLOMAVIRUS INFECTIONS AND ASSOCIATED RISK FACTORS IN MEN-WHO-HAVE-SEX-WITH-MEN IN CAPE TOWN, SOUTH AFRICA}

${ }^{1}$ EE Müller, ${ }^{2,3,4} \mathrm{~K}$ Rebe, ${ }^{5} \mathrm{TF}$ Chirwa, ${ }^{2,3,4} \mathrm{H}$ Struthers, ${ }^{2,3,6} \mathrm{~J}$ McIntyre, ${ }^{7,8} \mathrm{DA}$ Lewis. ${ }^{1}$ Centre for HIV and Sexually Transmitted Infections, National Institute for Communicable Diseases, National Health Laboratory Service, Johannesburg, South Africa; ${ }^{2}$ Anova Health Institute, Johannesburg, South Africa; ${ }^{3}$ Anova Health Institute, Cape Town, South Africa; ${ }^{4}$ Department of Medicine, Division of Infectious Diseases and HIV Medicine, University of Cape Town, Cape Town, South Africa; ${ }^{5}$ Division of Epidemiology and Biostatistics, School of Public Health, University of the Witwatersrand, Johannesburg, South Africa; ${ }^{6}$ Division of Epidemiology and Biostatistics, School of Public and Family Medicine, University of Cape Town, Cape Town, South Africa; ${ }^{7}$ Western Sydney Sexual Health Centre, Western Sydney Local Health District, Parramatta, Australia; ${ }^{8}$ Marie Bashir Institute for Infectious Diseases and Biosecurity and Sydney Medical School-Westmead, University of Sydney, Sydney, Australia

\subsection{6/sextrans-2017-053264.307}

Introduction We investigated the prevalence of high-risk (HR) human papillomavirus (HPV) infections and associated behavioural risk factors in men-who-have-sex-with-men (MSM) attending a clinical service in Cape Town, South Africa.

Methods MSM were enrolled at the Ivan Toms Centre for Men's Health in Cape Town. For each participant, a psychosocial and sexual behavioural risk questionnaire was completed and an anal swab was collected and tested for $13 \mathrm{HR}-\mathrm{HPV}$ types using the Linear Array HPV Genotyping Test. Logistic regression analyses were performed to determine sexual risk factors associated with anal HR-HPV infection.

Results The median age of the 200 participants was 32 years (IQR 26-39.5), of which 31.0\% were black, 31.5\% mixed race and $35.5 \%$ white. The majority of the participants (73.0\%) had completed high school, $42.0 \%$ had a tertiary level qualification and $69.0 \%$ were employed. The prevalence of HR-HPV types was $57.6 \%$ [95\% CI: 50.3\%-64.7\%] in anal samples, with HPV-16 being the most common HR-HPV type detected (22.0\%). HPV types 16 and/or 18, the only HR-HPV types included in all available HPV vaccines, were detected in $55(28.8 \%)$ [95\% CI: 22.5\%-35.8\%] anal specimens. Furthermore, 95 (49.7\%) [95\% CI: 42.9\%-57.1\%] specimens had one or more HR-HPV types included in the 9 valent HPV vaccine. Among the 88 MSM (44.0\%) that were HIV positive, $81.2 \%$ [95\% CI: $71.2 \%-88.8 \%$ ] had anal HR- 